\title{
Rapid Tomography in Environmental TEM: How Fast Can We Go to Follow the 3D Evolution of Nanomaterials in situ?
}

\author{
Lucian Roiban ${ }^{1}$, Sid Koneti ${ }^{1}$, Khan Tran $^{2}$, Yue-Meng Feng ${ }^{2}$, Thomas Grenier ${ }^{2}$, Voichita Maxim ${ }^{2}$ \\ and Thierry Epicier ${ }^{1}$ \\ 1. Univ Lyon, INSA-Lyon, Université Claude Bernard, CNRS UMR 5510, MATEIS, Bât. Blaise Pascal, \\ 69621 Villeurbanne, France. \\ 2. Univ. Lyon, INSA-Lyon, CNRS UMR 5220, Inserm U1044, CREATIS, Bât. Blaise Pascal, 69621 \\ Villeurbanne, France.
}

Environmental Transmission Electron Microscopy (ETEM) in a dedicated instrument has been the subject of recent considerable developments allowing to follow chemical reactions in situ under environmental, e.g. gas and temperature conditions even at atomic resolution. A typical domain of applications concerns catalysis, where supported nanoparticles (NPs) are followed during calcination, oxidation or reduction in the presence of various gases. Whereas numerous works have now been published in conventional imaging, that is, 2D projection, little work is reported on 3D investigations performed in situ. It is easy to understand why such an approach is difficult: since TEM tomography consists in reconstructing numerically a tilt series of projections over a wide angular range, the time to acquire these data is generally too important as compared to the speed of the sample evolution, or the kinetics of the studied chemical reaction. The duration of the conventional acquisition step is typically one hour, or a fraction of one hour, making it impossible to get tilting sequences where the object does not experience any significant shape change which obviously hampers 3D reconstruction.

This trivial statement leads to the conclusion that speeding up the acquisition procedure is the key of Environmental Tomography. This contribution summarizes where we are in developing Fast Tomography in Bright Field (E)TEM at the minute and even second level mainly for applications on nano-catalysts. Experiments are performed in a FEI Titan ETEM and using a Wildfire heating holder from DENS Solutions allowing $\pm 70^{\circ}$ tilt with S5-type MEMS-based $\mathrm{SiN}_{\mathrm{x}}$ chips.

Three levels of action are being undertaken to overcome problems and limitations regarding image sharpness (blur effects due to the sample rotation and motion) and SNR (short exposure time):

(i) Optimize the acquisition for either a step-by-step or a continuous rotation tilting procedure, the latter leading to the recording of video sequences instead of classical elementary images.

(ii) Decrease as much as possible the acquisition time, as allowed with the latest generation of cameras for electron microscopy (here a Gatan Oneview camera used in the $4 \mathrm{Kx} 4 \mathrm{~K}$ and $2 \mathrm{Kx} 2 \mathrm{~K}$ modes at respectively 40 and $10 \mathrm{~ms}$ of acquisition and 1 to 4 electron per pixel).

(iii) Improve the post mortem automatic treatment of acquired data in terms of sorting blurred and sufficient sharp images and deblurring routines of video sequences.

Present results, limits and perspectives regarding duration and final resolution will be illustrated through several examples on various catalytic systems (Figures below). Most experiments were conducted under a partial pressure of oxygen up to a few mbars at temperatures up to $800^{\circ} \mathrm{C}$. Acquisition of tilt series was in all cases shorter than 200 seconds, down to 7 seconds for the fastest one successfully reconstructed in 3D with a final resolution of a few nanometers. In several cases numerous tomographic reconstructions were performed on the same object experiencing different environmental conditions, thus opening the way to truly follow the 3D evolution of nanomaterials in operando as shown in Figure 2 [1]. 


\section{References:}

[1] This work is performed within the framework of the French ANR project $\mathrm{n}^{\circ} 15$-CE09-0009-01 "3DCLEAN" http://www.clym.fr/3DCLEAN_web/3DCLEAN-ANR.html). The authors thank the CLYM (Consortium Lyon-Saint Etienne de Microscopie, www.clym.fr) for the access to the microscope and A. Paczad (Gatan USA) for the loan of the Oneview camera. Colleagues having provided samples are gratefully acknowledged: A.S. Gay from IFPen (Solaize, F) for $\mathrm{Pd}_{0} \mathrm{Al}_{2} \mathrm{O}_{3}$, A. Serve and P. Vernoux from Ircelyon (Villeurbanne, F) for soot@ $\mathrm{ZrO}_{2}$. M. Aouine (Ircelyon) and F. Dalmas (MATEIS) are thanked for useful discussions and experimental contributions to this work.
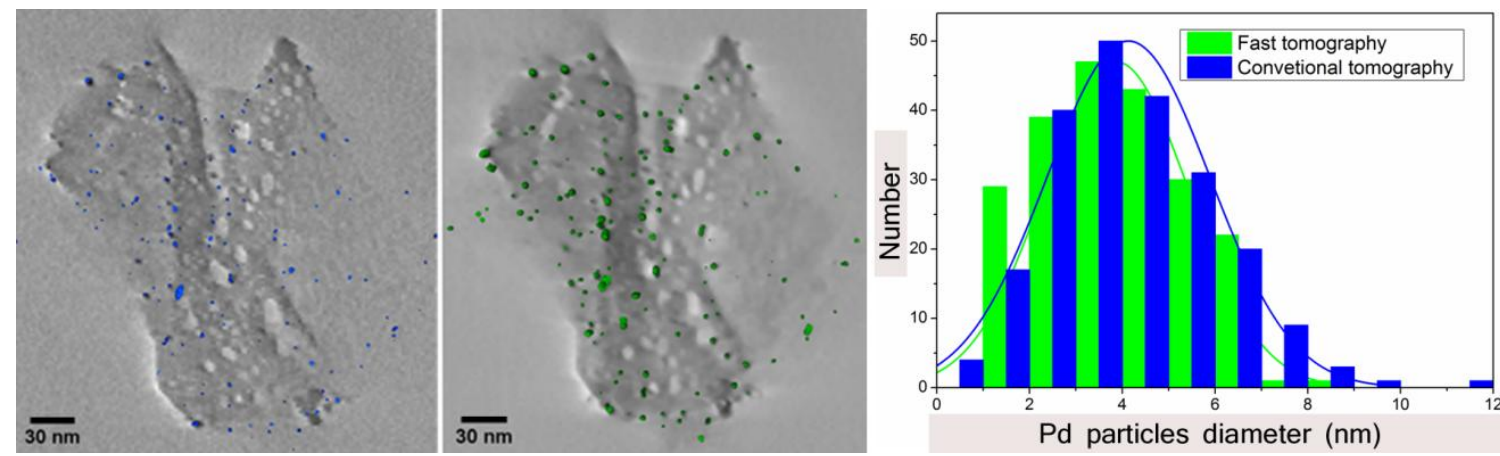

Figure 1. TEM reconstructed volumes of $\delta$-alumina supporting Pd NPs (shown in color): left: conventional bright field tomography acquired in 45 minutes; middle: fast tomography sequence acquired in 150 seconds with the continuous tilt approach (both sequences recorded under high vacuum over an angular amplitude -74 to $66^{\circ}$ ). Right: comparison of the very similar Pd particle size 3D measurements (respectively 4.1 and $3.9 \mathrm{~nm}$ for the conventional and fast tomography).

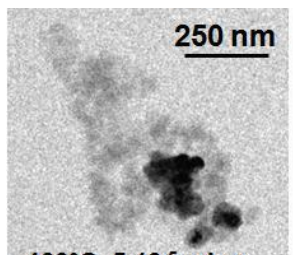

$400^{\circ} \mathrm{C}, 510^{-5} \mathrm{mbar}$

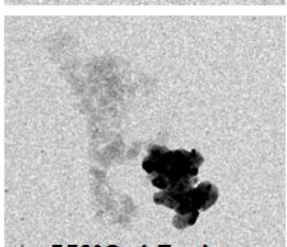

$550^{\circ} \mathrm{C}, 1.7 \mathrm{mbar}$

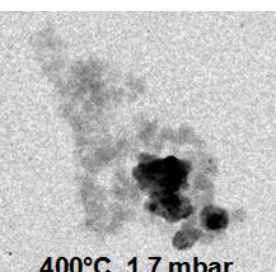

$400^{\circ} \mathrm{C}, 1.7 \mathrm{mbar}$

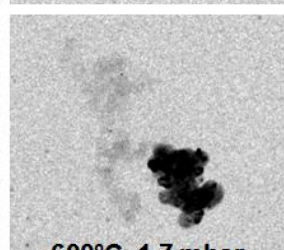

$600^{\circ} \mathrm{C}, 1.7 \mathrm{mbar}$
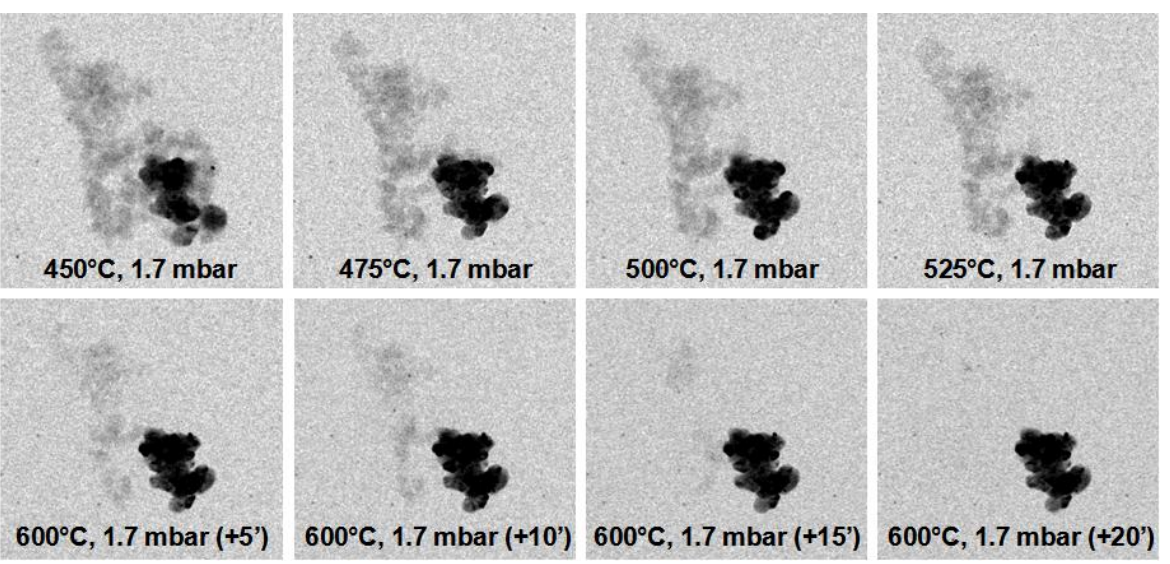

Figure 2. ETEM tomography series to follow the consumption of soot on $\mathrm{ZrO}_{2}$-based nano-catalysts under oxygen between $400^{\circ} \mathrm{C}$ and $600^{\circ} \mathrm{C}$ as indicated. Each $4 \mathrm{Kx} 4 \mathrm{~K}$ image exposed 0.04 sec is the zerotilt projection of an optimized step-by-step tilted series of the same object recorded between 72 and $70^{\circ} \mathrm{C}$ in 129 seconds during a heating ramp of $1 \% \mathrm{~min}$. At each temperature, 5 tomography series were recorded every 5 minutes (see the last 5 images) leading to a total of 35 series recorded over a time amplitude of $3 \mathrm{~h} 40$. During this interval, the sample was exposed to the electron beam only during the acquisitions for a total time of $1 \mathrm{~h} 45$ with a total electron dose of $2.910^{4} \mathrm{e}-/ \AA^{2}$. An accelerated irradiation test performed with the same electron dose at $300^{\circ} \mathrm{C}$ (to avoid any soot combustion) did not reveal any detectable morphological change of the microstructure. 